\title{
Area Spectral Efficiency and Energy Efficiency Tradeoff in Ultradense Heterogeneous Networks
}

\author{
Lanhua Xiang, Hongbin Chen, and Feng Zhao \\ Key Laboratory of Cognitive Radio and Information Processing, Guilin University of Electronic Technology, Guilin 541004, China \\ Correspondence should be addressed to Hongbin Chen; chbscut@guet.edu.cn
}

Received 4 March 2017; Accepted 15 June 2017; Published 12 July 2017

Academic Editor: Bernard Cousin

Copyright (C) 2017 Lanhua Xiang et al. This is an open access article distributed under the Creative Commons Attribution License, which permits unrestricted use, distribution, and reproduction in any medium, provided the original work is properly cited.

\begin{abstract}
In order to meet the demand of explosive data traffic, ultradense base station (BS) deployment in heterogeneous networks (HetNets) as a key technique in $5 \mathrm{G}$ has been proposed. However, with the increment of BSs, the total energy consumption will also increase. So, the energy efficiency (EE) has become a focal point in ultradense HetNets. In this paper, we take the area spectral efficiency (ASE) into consideration and focus on the tradeoff between the ASE and EE in an ultradense HetNet. The distributions of BSs in the two-tier ultradense HetNet are modeled by two independent Poisson point processes (PPPs) and the expressions of ASE and EE are derived by using the stochastic geometry tool. The tradeoff between the ASE and EE is formulated as a constrained optimization problem in which the EE is maximized under the ASE constraint, through optimizing the BS densities. It is difficult to solve the optimization problem analytically, because the closed-form expressions of ASE and EE are not easily obtained. Therefore, simulations are conducted to find optimal BS densities.
\end{abstract}

\section{Introduction}

The past decade has witnessed a rapid development in mobile communication networks, owing to the increasing number of new mobile communication devices, which led to an exponential growth in the wireless traffic volume and the surge of access points. It was predicted that, in future wireless networks, the data traffic density will increase 1000 times; at the same time, BS density will reach 10 times more than the density of the existing sites $[1,2]$. In order to achieve these goals, ultradense HetNet was considered as one of the key techniques to fulfill the future network requirements. A HetNet consists of various low-power small cells, such as micro-, pico-, and femto-BSs, which overlay the traditional macro cells. Then increasing the network density at different tiers in a HetNet can constitute an ultradense HetNet. Nevertheless, with the ultradense deployment of BSs, the energy consumption and $\mathrm{CO}_{2}$ emission will inevitably increase. Reference [3] investigated how much energy is needed to run a wireless network. The current study showed that the amount of $\mathrm{CO}_{2}$ emission by information and communication technologies exceeds 2\% [4]. As such, it is imperative that "green communication" be introduced and enforced to harness the environmental pollution and curb energy consumption. So, improving the $\mathrm{EE}$ of ultradense HetNets will be a significant part in the future research accompanying the proposed idea in 5G, namely, high EE, low latency, and seamless coverage. However, the traditional study on cellular networks primarily considered the SE instead of the EE. And the existing study on ultradense networks mainly focused on the ASE and EE in homogeneous networks rather than heterogeneous networks $[5,6]$.

In order to improve the $\mathrm{EE}$, on one hand, since BSs are major energy consumers in cellular networks, zooming [7] and BS sleep scheduling [8-10] were seen to be promising ways to reduce the energy consumption; on the other hand, introducing small cells into existing macro cell networks is also an effective technique [11]. However, sleep scheduling which is a technique in the media access control (MAC) layer actually is not easy to handle in the physical layer. So, this work tries to maximize the network EE following the second method by optimizing the BS deployment and determining optimal BS density. Earlier researches [12,13] paid attention to the BS density optimization for energy saving in ultradense networks. Recently, the authors of [14] discussed the spectral and energy efficiency of ultradense networks under different 
deployment strategies via strict simulations. These studies laid a good foundation on the BS density optimization for energy saving but mainly depended on simulations without rigorous theoretical analysis.

Stochastic geometry is a powerful mathematical tool for modeling, analysis, and design of wireless networks from earliest ad hoc wireless networks [15] to latest multitier and cognitive cellular wireless networks $[16,17]$. References [1822] summarized and compared many kinds of point processes and concluded that Poisson point process (PPP) is the most popular, most tractable, and most important one because of its independence property. Therefore, taking advantage of the PPP, we adopt the stochastic geometry theory to analyze the EE in an ultradense HetNet.

Though the previous works have studied the EE of ultradense networks, maximizing the EE under the constraints on ASE in ultradense HetNets has not been fully addressed. Therefore, in this paper, by modeling the BSs and mobile users (MUs) as independent spatial PPPs, the ASE and EE of downlink transmission in a two-tier ultradense HetNet are considered. In general, in many earlier works, the ASE and EE are defined as the amount of transmitted bps per unit bandwidth and the amount of transmitted bits per unit energy consumption, respectively. Different from previous definitions, in this work the ASE is defined as the area total throughout per unit bandwidth per unit area, and the EE is defined as the ratio of area total throughout to area total power consumption. Moreover, interference is also an important factor influencing the ASE and EE that cannot be ignored, especially in the scenario of ultradense HetNets. The contributions of this paper are summarized as follows. Firstly, taking the intertier interference and intratier interference into consideration, we analyze the SINR of MUs and the total throughout concerning the point density and the BS transmit power. Secondly, according to the definitions of ASE and EE, we obtain the analytical expressions of the networks ASE and EE and then formulate the optimization problem to balance the ASE and EE. Finally, as it is difficult to solve the optimization problem analytically, we find out the optimal ratios of BS density to user density by simulations.

The remainder of this paper is organized as follows. In Section 2, the model of downlink transmission in a two-tier ultradense HetNet with the PPP modeling is described. Theoretical analysis of the ASE and EE by stochastic geometry is deduced in Section 3, together with the optimization problem of balancing the ASE and EE. Simulation results are illustrated in Section 4. Finally, conclusions are given in Section 5.

\section{System Model}

A two-tier downlink ultradense HetNet consisting of macro cell base stations ( $\mathrm{mBSs}$ ) and picrocell base stations ( $\mathrm{pBSs}$ ) is considered in an area $\dot{A}$, where the BSs are distributed according to two independent homogeneous PPPs $\Phi_{k}(k=1,2)$ with densities $\lambda_{k}$ in the two-dimensional Euclidean plane. Since the transmit powers of BSs across tiers are different, the coverage regions of BSs form a weighted Poisson Voronoi Tessellation (PVT). Figure 1 shows an example of coverage region of the two-tier downlink ultradense HetNet.

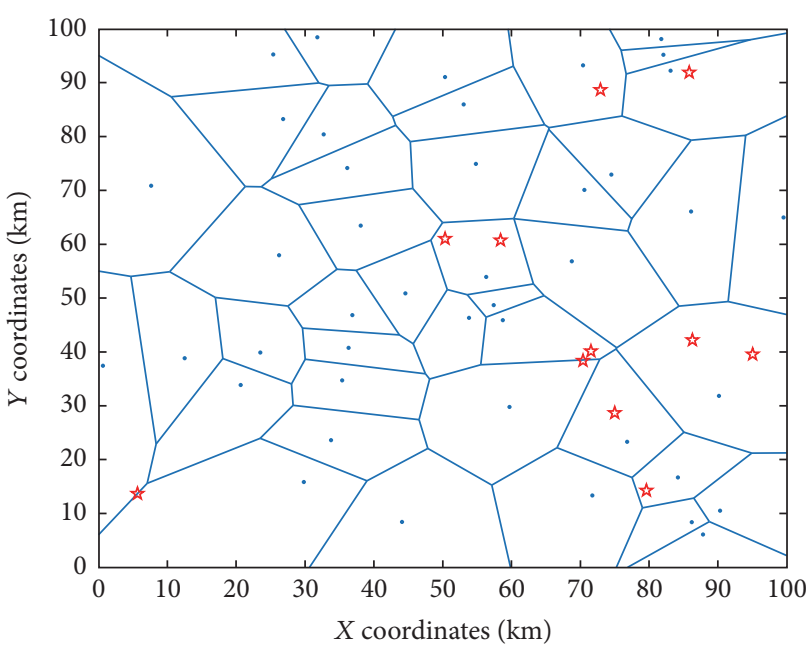

Figure 1: An example of PVT for the two-tier downlink ultradense HetNet including mBSs (pentagons, $k=1$ ) and pBSs (dots, $k=2$ ), $\lambda_{2}=3 \lambda_{1}$.

The MUs are also modeled by an independent PPP $\Phi_{u}$ of the density $\lambda_{u}$. In order to describe the influence of BS deployment on the ASE and EE more clearly, here the BS density is normalized as $\lambda_{k}=\Delta_{k} \lambda_{u}$, where $\Delta_{k}$ is defined as the ratio of number of BSs to number of MUs in $k$ th tier.

The transmit power of $k$ th tier BS is $P_{k}$. We consider the universal frequency reuse over the total available bandwidth $B$. Without loss of generality, we conduct the analysis on a typical MU located at the origin and the MU is named as tagged MU. Each MU will associate with a closest BS. The channel between the tagged MU and its serving BS is modeled as a Rayleigh fading channel with mean 1 on all links in $k$ th tier, which is denoted by $h_{k}$, that is, $h_{k} \sim \exp (1)$. The path loss exponent is represented by $\alpha(>2)$. The channel noise is an additive white Gaussian noise with zero mean and variance $\delta^{2}$. Except for the serving BS of the tagged MU, all other BSs in the ultradense HetNet are regarded as interfering BSs. The distance between $i$ thBS of $k$ th tier and the tagged MU is $R_{k, i}$, and the corresponding channel fading is $H_{k, i}$. Similar to $h_{k}$, $H_{k, i} \sim \exp (1)$. Therefore, according to Shannon's law, the SINR of a typical MU associating with its serving BS at the distance of $r_{k}$ can be expressed as

$$
\operatorname{SINR}\left(r_{k}\right)=\frac{P_{k} h_{k} r_{k}^{-\alpha}}{I+\delta^{2}},
$$

where $I$ is the total interference power received by the tagged $\mathrm{MU}$ from interfering BSs, $I=\sum_{k=1}^{2} I_{k}, I_{k}=$ $\sum_{i \in \Phi_{k} / b_{k}} P_{k} H_{k, i} R_{k, i}{ }^{-\alpha}$, and $b_{k}$ is the serving BS of the tagged MU.

\section{Analysis of Network Performance}

3.1. Coverage Probability. The coverage probability of BSs in $k$ th tier is defined as the probability that a randomly chosen MU can achieve a target $\operatorname{SINR} \theta_{k}$ when associating with a BS 
in $k$ th tier (or equivalent as the average fraction of MUs who achieve a target SINR $\theta_{k}$ when associating with a BS in $k$ th tier). We first derive the coverage probability of the mBSs as [16]

$$
\begin{aligned}
p & {\left[\operatorname{SINR}\left(r_{1}\right)>\theta_{1}\right]=p\left[\frac{P_{1} h_{1} r_{1}^{-\alpha}}{I+\delta^{2}}>\theta_{1}\right] } \\
& =p\left[h_{1}>\left(I+\delta^{2}\right) \frac{\theta_{1} r_{1}^{\alpha}}{P_{1}}\right] \\
& =E_{I}\left[\int_{\left(I+\delta^{2}\right)\left(\theta_{1} r_{1}{ }^{\alpha} / P_{1}\right)}^{\infty}[\exp (-x)] \mathrm{d} x\right] \\
& =E_{I}\left[\exp \left[-\left(I+\delta^{2}\right) \frac{\theta_{1} r_{1}^{\alpha}}{P_{1}}\right]\right],
\end{aligned}
$$

where the penultimate step is derived by noting that $h_{k}$ is an exponential random variable. Because the $\mathrm{mBS}$ s and $\mathrm{pBS}$ are two independent homogeneous PPPs and the interference brought by them is also independent, we get

$$
\begin{aligned}
p[ & \left.\operatorname{SINR}>\theta_{1}\right] \\
& =e^{-\left(\theta_{1} r_{1}^{\alpha} / P_{1}\right) \delta^{2}} E_{I_{1}}\left(e^{-\left(\theta_{1} r_{1}^{\alpha} / P_{1}\right) I_{1}}\right) E_{I_{2}}\left(e^{-\left(\theta_{1} r_{1}^{\alpha} / P_{1}\right) I_{2}}\right) \\
& =e^{-\left(\theta_{1} r_{1}^{\alpha} / P_{1}\right) \delta^{2}} L_{I_{1}}\left(\frac{\theta_{1} r_{1}^{\alpha}}{P_{1}}\right) L_{I_{2}}\left(\frac{\theta_{1} r_{1}^{\alpha}}{P_{1}}\right)
\end{aligned}
$$

where $L_{I_{1}}\left(s_{1}\right)$ and $L_{I_{2}}\left(s_{1}\right)$ are Laplace transforms of random variables $I_{1}$ and $I_{2}$ evaluated at $s_{1}\left(s_{1}=\theta_{1} r_{1}{ }^{\alpha} / P_{1}\right)$, respectively. Therefore, the averaged coverage probability of mBSs over the plane is derived as

$$
\begin{aligned}
p_{1} & =E_{r_{1}}\left[p\left[\operatorname{SINR}>\theta_{1}\right]\right] \\
& =\int_{0}^{\infty}\left[p\left[\operatorname{SINR}>\theta_{1}\right]\right] f_{r}\left(r_{1}\right) \mathrm{d} r_{1},
\end{aligned}
$$

where $f_{r}\left(r_{1}\right)=e^{-\pi \lambda_{1} r_{1}^{2}} 2 \pi \lambda_{1} r_{1}$ is the probability density function of $r_{1}$. As mentioned before, $r_{1}$ is the random distance between the tagged macro MU and its corresponding serving mBS. Then,

$$
\begin{aligned}
p_{1} & =E_{r_{1}}\left[p\left[\operatorname{SINR}>\theta_{1}\right]\right] \\
& =\int_{0}^{\infty} e^{-\left(\theta_{1} r_{1}{ }^{\alpha} / P_{1}\right) \delta^{2}} L_{I_{1}}\left(\frac{\theta_{1} r_{1}^{\alpha}}{P_{1}}\right) L_{I_{2}}\left(\frac{\theta_{1} r_{1}^{\alpha}}{P_{1}}\right) \\
& \cdot e^{-\pi \lambda_{1} r_{1}{ }^{2}} 2 \pi \lambda_{1} r_{1} \mathrm{~d} r_{1} .
\end{aligned}
$$

From the definition of the Laplace transform, we calculate the Laplace transform of $I_{1}$ which is the aggregate interference power generated by the mBSs at the tagged macro MU as follows:

$$
\begin{aligned}
L_{I_{1}}\left(s_{1}\right) & =E_{I_{1}}\left[e^{-s_{1} I_{1}}\right]=E_{\Phi_{1}, H_{1, i}}\left[e^{-e s_{1} \sum_{i \in \Phi_{1} / b_{1}} P_{1} H_{1, i} R_{1, i}-\alpha}\right] \\
& =E_{\Phi_{1}}\left[\prod_{i \in \Phi_{1} / b_{1}} E_{H_{1, i}}\left(e^{-s_{1} P_{1} H_{1, i} R_{1, i}^{-\alpha}}\right)\right] \\
& =e^{-2 \pi \lambda_{1} \int_{r_{1}}^{\infty}\left(1-\int_{0}^{\infty} e^{-s_{1} P_{1} H_{1} R_{1}^{-\alpha}} e^{-H_{1}} \mathrm{~d} H_{1}\right) R_{1} \mathrm{~d} R_{1}} \\
& =e^{-2 \pi \lambda_{1} \int_{r_{1}}^{\infty}\left(1-\mu_{1} /\left(s_{1} R_{1}^{-\alpha}+\mu_{1}\right)\right) R_{1} \mathrm{~d} R_{1}}
\end{aligned}
$$

where $\mu_{1}=1 / P_{1}$; the antepenultimate step is derived by noting that $H_{k, i}$ are i.i.d. distributed and its further independence from the point process $\Phi_{k}$; the penultimate step is obtained according to the probability generating function of PPP $[23,24]$, which states for some function $f(x)$ that $E\left[\prod_{x \in \Phi} f(x)\right]=\exp \left(-\lambda \int_{R^{2}}(1-f(x)) \mathrm{d} x\right)$. Since the closest interfering $\mathrm{mBS}$ is at least at the distance $r_{1}$ from the tagged macro MU, the integration limits are from $r_{1}$ to $\infty$. Plugging in $s_{1}\left(s_{1}=\mu_{1} \theta_{1} r_{1}{ }^{\alpha}\right)$, we get

$$
\begin{aligned}
& 2 \pi \lambda_{1} \int_{r_{1}}^{\infty}\left(1-\frac{\mu_{1}}{s_{1} R_{1}^{-\alpha}+\mu_{1}}\right) R_{1} \mathrm{~d} R_{1} \\
& =2 \pi \lambda_{1} \int_{r_{1}}^{\infty}\left(1-\frac{\mu_{1}}{\mu_{1} r_{1}^{\alpha} \theta_{1} R_{1}^{-\alpha}+\mu_{1}}\right) R_{1} \mathrm{~d} R_{1} \\
& =2 \pi \lambda_{1} \int_{r_{1}}^{\infty}\left(1-\frac{1}{r_{1}^{\alpha} \theta_{1} R_{1}{ }^{-\alpha}+1}\right) R_{1} \mathrm{~d} R_{1} \\
& =2 \pi \lambda_{1} \int_{r_{1}}^{\infty}\left(\frac{r_{1}^{\alpha} \theta_{1} R_{1}^{-\alpha}}{r_{1}{ }^{\alpha} \theta_{1} R_{1}^{-\alpha}+1}\right) R_{1} \mathrm{~d} R_{1} \\
& =2 \pi \lambda_{1} \int_{r_{1}}^{\infty}\left(\frac{1}{r_{1}{ }^{-\alpha} R_{1}^{\alpha} / \theta_{1}+1}\right) R_{1} \mathrm{~d} R_{1} \\
& =2 \pi \lambda_{1} \int_{r_{1}}^{\infty}\left(\frac{1}{1+\left[r_{1}^{-1} R_{1} / \theta_{1}^{1 / \alpha}\right]^{2 \cdot(\alpha / 2)}}\right) R_{1} \mathrm{~d} R_{1} \\
& =\pi \lambda_{1} r_{1}^{2} \theta_{1}^{2 / \alpha} \int_{\theta_{1}{ }^{-2 / \alpha}}^{\infty}\left(\frac{1}{1+x^{\alpha / 2}}\right) \mathrm{d} x\left(x=\left[r_{1}{ }^{-1} R_{1} / \theta_{1}^{1 / \alpha}\right]^{2}\right) .
\end{aligned}
$$

Taking (7) into (6) and letting $s_{1}\left(s_{1}=\theta_{1} r_{1}{ }^{\alpha} / P_{1}\right),(6)$ can be finally expressed as

$$
L_{I_{1}}\left(\frac{\theta_{1} r_{1}^{\alpha}}{P_{1}}\right)=e^{-\pi \lambda_{1} r_{1}^{2} \theta_{1}^{2 / \alpha} \int_{\theta_{1}-2 / \alpha}^{\infty}\left(1 /\left(1+x^{2 / \alpha}\right)\right) \mathrm{d} x} .
$$


In this way, the Laplace transform of $I_{2}$ which is the aggregate interference power generated by the pBSs at the tagged macro $\mathrm{MU}$ is given by

$$
\begin{aligned}
L_{I_{2}}\left(s_{1}\right) & =E_{I_{2}}\left[e^{-s_{1} I_{2}}\right]=E_{\Phi_{2}, H_{2, i}}\left[e^{-e s_{1} \sum_{i \in \Phi_{2}} P_{2} H_{2, i} R_{2, i}-\alpha}\right] \\
& =E_{\Phi_{2}}\left[\prod_{i \in \Phi_{2}} E_{H_{2, i}}\left(e^{-s_{1} P_{2} H_{2, i} R_{2, i}-\alpha}\right)\right] \\
& =e^{-2 \pi \lambda_{2} \int_{0}^{\infty}\left(1-\int_{0}^{\infty} e^{-s_{1} P_{2} H_{2} R_{2}-\alpha} e^{-H_{2}} \mathrm{~d} H_{2}\right) R_{2} \mathrm{~d} R_{2}} \\
& =e^{-2 \pi \lambda_{2} \int_{0}^{\infty}\left(1-\mu_{2} /\left(s_{1} R_{2}^{-\alpha}+\mu_{2}\right)\right) R_{2} \mathrm{~d} R_{2}}\left(\mu_{2}=\frac{1}{P_{2}}\right) .
\end{aligned}
$$

Since the interference to the tagged macro $\mathrm{MU}$ is encountered from all pBSs, the integration limits are from 0 to $\infty$. Then, plugging in $s_{1}\left(s_{1}=\mu_{1} \theta_{1} r_{1}{ }^{\alpha}\right)$, we get

$$
\begin{aligned}
& 2 \pi \lambda_{2} \int_{0}^{\infty}\left(1-\frac{\mu_{2}}{s_{1} R_{2}^{-\alpha}+\mu_{2}}\right) R_{2} \mathrm{~d} R_{2} \\
& =2 \pi \lambda_{2} \int_{0}^{\infty}\left(1-\frac{\mu_{2}}{\mu_{1} r_{1}{ }^{\alpha} \theta_{1} R_{2}^{-\alpha}+\mu_{2}}\right) R_{2} \mathrm{~d} R_{2}
\end{aligned}
$$

$$
\begin{aligned}
& =2 \pi \lambda_{2} \int_{0}^{\infty}\left(1-\frac{1}{\beta_{1} r_{1}^{\alpha} \theta_{1} R_{2}^{-\alpha}+1}\right) R_{2} \mathrm{~d} R_{2} \\
& =2 \pi \lambda_{2} \int_{0}^{\infty}\left(\frac{\beta_{1} r_{1}^{\alpha} \theta_{1} R_{2}^{-\alpha}}{\beta_{1} r_{1}{ }^{\alpha} \theta_{1} R_{2}{ }^{-\alpha}+1}\right) R_{2} \mathrm{~d} R_{2} \\
& =2 \pi \lambda_{2} \int_{0}^{\infty}\left(\frac{1}{r_{1}{ }^{-\alpha} R_{2}{ }^{\alpha} / \beta_{1} \theta_{1}+1}\right) R_{2} \mathrm{~d} R_{2} \\
& =2 \pi \lambda_{2} \int_{0}^{\infty}\left(\frac{1}{1+\left[r_{1}{ }^{-1} R_{2} /\left(\beta_{1} \theta_{1}\right)^{1 / \alpha}\right]^{2 \cdot(\alpha / 2)}}\right) R_{2} \mathrm{~d} R_{2} \\
& =\pi \lambda_{2} r_{1}{ }^{2} \beta_{1}{ }^{2 / \alpha} \theta_{1}{ }^{2 / \alpha} \int_{0}^{\infty}\left(\frac{1}{1+y^{\alpha / 2}}\right) \mathrm{d} y\left(y=\left[\frac{r_{1}{ }^{-1} R_{2}}{\left(\beta_{1} \theta_{1}\right)^{1 / \alpha}}\right]^{2}\right),
\end{aligned}
$$

where $\beta_{1}=\mu_{1} / \mu_{2}$.

By substituting (10) into (9) and letting $s_{1}\left(s_{1}=\theta_{1} r_{1}{ }^{\alpha} / P_{1}\right)$, (9) can be finally expressed as

$$
L_{I_{2}}\left(\frac{\theta_{1} r_{1}^{\alpha}}{P_{1}}\right)=e^{-\pi \lambda_{2} r_{1}^{2} \beta_{1}^{2 / \alpha} \theta_{1}^{2 / \alpha} \int_{0}^{\infty}\left(1 /\left(1+y^{2 / \alpha}\right)\right) \mathrm{d} y} .
$$

Substituting (8) and (11) into (5), we can obtain the averaged coverage probability of $\mathrm{mBSs}$ over the plane as follows:

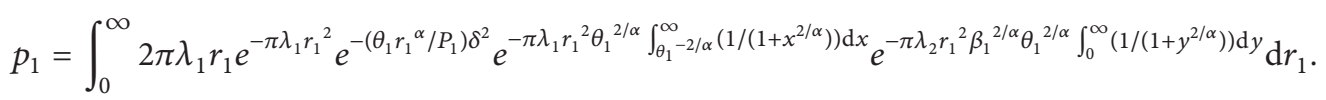

Same as the above derivation, the averaged coverage probability of pBS over the plane is derived as

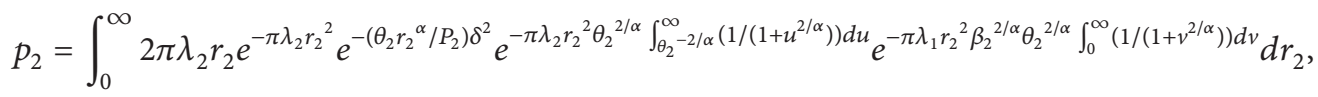

where $\beta_{2}=\mu_{2} / \mu_{1}$.

3.2. Average Achievable Rate. The average achievable rate by a randomly chosen $\mathrm{MU}$ when it is under coverage of BSs in $k$ th tier can be expressed as

$$
R_{k}=\frac{B}{N_{k}} \mathbb{E}_{r_{k}}\left[\log _{2}\left(1+\operatorname{SINR}\left(r_{k}\right)\right) \mid \operatorname{SINR}\left(r_{k}\right)>\theta_{k}\right],
$$

where $N_{k}$ is the average number of MUs served by the tagged $\mathrm{BS}$ in $k$ th tier. Based on Corollary 1 in [25], we can obtain

$$
N_{k}=1+1.28 \frac{\lambda_{u} p_{k}}{\lambda_{k}}=1+1.28 \frac{p_{k}}{\Delta_{k}} .
$$

We first derive the conditional expectation as follows:

$$
\begin{aligned}
\mathbb{E}_{r_{k}} & {\left[\log _{2}\left(1+\operatorname{SINR}\left(r_{k}\right)\right) \mid \operatorname{SINR}\left(r_{k}\right)>\theta_{k}\right] } \\
\quad= & \mathbb{E}_{r_{k}}\left[\int_{0}^{\infty} \log _{2}(1+Y) f_{Y}\left(y \mid Y>\theta_{k}\right) \mathrm{d} y\right](Y
\end{aligned}
$$

$$
\begin{aligned}
& \left.=\operatorname{SINR}\left(r_{k}\right)\right)=\frac{1}{\ln 2} \\
& \cdot \mathbb{E}_{r_{k}}\left[\int_{0}^{\infty} \int_{0}^{Y} \frac{1}{(z+1)} f_{Y}\left(y \mid Y>\theta_{k}\right) \mathrm{d} z \mathrm{~d} y\right] \\
& =\frac{1}{\ln 2} \\
& \cdot \mathbb{E}_{r_{k}}\left[\int_{0}^{\infty}\left(\int_{z}^{\infty} f_{Y}\left(y \mid Y>\theta_{k}\right) \mathrm{d} y\right) \frac{1}{z+1} \mathrm{~d} z\right] \\
& =\frac{1}{\ln 2} \mathbb{E}_{r_{k}}\left[\int_{0}^{\infty} \frac{p\left(Y>z \mid Y>\theta_{k}\right)}{z+1} \mathrm{~d} z\right]=\frac{1}{\ln 2} \\
& \cdot \int_{0}^{\infty}\left(\int_{0}^{\infty} \frac{p\left(Y>z \mid Y>\theta_{k}\right)}{z+1} \mathrm{~d} z\right) f\left(r_{k}\right) \mathrm{d} r_{k}
\end{aligned}
$$




$$
\begin{aligned}
& =\frac{1}{\ln 2} \int_{0}^{\infty}\left(\int_{0}^{\infty} \frac{p\left(Y>z \mid Y>\theta_{k}\right)}{z+1} \mathrm{~d} z\right) \\
& \cdot 2 \pi \lambda_{k} r_{k} e^{-\lambda_{k} \pi r_{k}^{2}} \mathrm{~d} r_{k} .
\end{aligned}
$$

Using Bayes' theorem, we can calculate the conditional probability in (16) as

$$
\begin{aligned}
& p\left(Y>z \mid Y>\theta_{k}\right)=\frac{p\left(Y>z, Y>\theta_{k}\right)}{p\left(Y>\theta_{k}\right)} \\
& =\frac{p\left(Y>\max \left(z, \theta_{k}\right)\right)}{p\left(Y>\theta_{k}\right)} \\
& = \begin{cases}\frac{L_{I_{1}}\left(z r_{k}^{\alpha} / P_{k}\right) L_{I_{2}}\left(z r_{k}^{\alpha} / P_{k}\right) e^{-\left(z r_{k}{ }^{\alpha} / P_{k}\right) \delta^{2}}}{L_{I_{1}}\left(\theta_{k} r_{k}^{\alpha} / P_{k}\right) L_{I_{2}}\left(\theta_{k} r_{k}^{\alpha} / P_{k}\right) e^{-\left(\theta_{k} r_{k}{ }^{\alpha} / P_{k}\right) \delta \delta^{2}}} & z>\theta_{k} \\
1 ; & \text { otherwise. }\end{cases}
\end{aligned}
$$

From (14)-(17), the expression for the average achievable rate by a randomly chosen MU when it is under coverage of BSs in $k$ th tier is obtained as

$$
\begin{aligned}
R_{k} & =\frac{B}{N_{k}} \mathbb{E}_{r_{k}}\left[\log _{2}\left(1+\operatorname{SINR}\left(r_{k}\right)\right) \mid \operatorname{SINR}\left(r_{k}\right)>\theta\right] \\
& =\frac{B}{N_{k}}\left(\log _{2}\left(1+\theta_{k}\right)+\frac{1}{\ln 2} \int_{0}^{\infty} \mathscr{A}\left(r_{k}\right) \mathrm{d} r_{k}\right),
\end{aligned}
$$

where

$$
\begin{aligned}
& \mathscr{A}\left(r_{k}\right)=2 \pi \lambda_{k} \int_{0}^{\infty} r_{k} \\
& \cdot \frac{e^{-\left(z r_{k}{ }^{\alpha} / P_{k}\right) \delta^{2}} L_{I_{1}}\left(z r_{k}^{\alpha} / P_{k}\right) L_{I_{2}}\left(z r_{k}^{\alpha} / P_{k}\right)}{e^{-\left(\theta_{k} r_{k}^{\alpha} / P_{k}\right) \delta^{2}} L_{I_{1}}\left(\theta_{k} r_{k}{ }^{\alpha} / P_{k}\right) L_{I_{2}}\left(\theta_{k} r_{k}{ }^{\alpha} / P_{k}\right)} \frac{1}{z+1} e^{-\lambda_{k} \pi r_{k}^{2}} \mathrm{~d} z .
\end{aligned}
$$

3.3. Total Throughput. We define total network throughput in $k$ th tier as the product of the total number of MUs served by the tagged BS in $k$ th tier and the average achievable rate by a randomly chosen MU when it is under coverage of BS in $k$ th tier. So, the total network throughput is expressed as

$$
T_{\text {total }}=p_{1} \lambda_{u} S_{\dot{A}} R_{1}+p_{2} \lambda_{u} S_{\dot{A}} R_{2},
$$

where $S_{\dot{A}}$ is the area of $\dot{A}$.

3.4. Total Power Consumption. The total power consumption of the two-tier downlink ultradense HetNet is given by [4]

$$
P_{\text {total }}=\lambda_{1} S_{\dot{A}}\left(p_{c 1}+\omega_{1} P_{1}\right)+\lambda_{2} S_{\dot{A}}\left(p_{c 2}+\omega_{2} P_{2}\right),
$$

where $p_{c k}$ is the static power consumption of BSs in $k$ th tier, which is used for signal processing and battery backup, together with site cooling, and is irrelevant with the BS transmit power; $\omega_{k}$ is the slope of the load-dependent power consumption of BSs in $k$ th tier. We set $\omega_{k}=N_{k}$.

3.5. Area Spectral Efficiency and Energy Efficiency Tradeoff. The ASE and EE are key metrics in the evaluation of network performance. According to the definition of ASE and EE in the introduction, they can be expressed as

$$
\begin{aligned}
\eta_{\text {ASE }} & =\frac{T_{\text {total }}}{B S_{\dot{A}}}=\frac{p_{1} \lambda_{u} S_{\dot{A}} R_{1}+p_{2} \lambda_{u} S_{\dot{A}} R_{2}}{B S_{\dot{A}}} \\
& =\frac{p_{1} \lambda_{u} R_{1}+p_{2} \lambda_{u} R_{2}}{B}, \\
\eta_{\mathrm{EE}} & =\frac{T_{\text {total }}}{P_{\text {total }}} \\
& =\frac{p_{1} \lambda_{u} S_{\dot{A}} R_{1}+p_{2} \lambda_{u} S_{\dot{A}} R_{2}}{\lambda_{1} S_{\dot{A}}\left(p_{c 1}+\omega_{1} P_{1}\right)+\lambda_{2} S_{\dot{A}}\left(p_{c 2}+\omega_{2} P_{2}\right)} \\
& =\frac{p_{1} \lambda_{u} R_{1}+p_{2} \lambda_{u} R_{2}}{\lambda_{1}\left(p_{c 1}+\omega_{1} P_{1}\right)+\lambda_{2}\left(p_{c 2}+\omega_{2} P_{2}\right)} .
\end{aligned}
$$

Based on the above analysis, we cannot derive the explicit mathematical expressions of ASE and EE. Then, we formulate an optimization problem to balance ASE and EE, namely, to maximize the EE under the ASE constraint. This optimization problem can be formulated as

$$
\begin{aligned}
\max _{P_{1}, P_{2}, \lambda_{1}, \lambda_{2}} & \eta_{\mathrm{EE}} \\
\text { s.t. } & \eta_{\mathrm{ASE}} \geq \bar{\eta}_{\mathrm{ASE}} \geq 0, \\
& 0 \leq P_{2}<P_{1} \leq P_{\max }, \\
& 0<\lambda_{1}<\lambda_{2},
\end{aligned}
$$

where $\bar{\eta}_{\mathrm{ASE}}$ is the minimum required ASE, $P_{\max }$ is maximum transmit power of BSs, the second constraint means that the transmit power of mBSs is more than the transmit power of pBSs but they cannot exceed the maximum transmit power of BSs, and the last constraint means that the mBS density is less than the $\mathrm{pBS}$ density.

In this work, we focus on the impact of BS density on the tradeoff between ASE and EE when transmit powers of BSs are fixed. According to (22), we can infer that if the BS density in each tier is set properly, maximum EE under the ASE constraint will be achieved. But, it is difficult to solve the optimization problem analytically, because the closed-form expressions of ASE and EE are not easily obtained. Therefore, in the following section, extensive simulations are executed to find out the optimal BS densities.

\section{Numerical Results}

In this section, we will validate the results of the above analysis through comparison of Monte Carlo simulation results and analytical results. The simulation parameters are listed in Table 1.

Figures 2 and 3 depict the relationship between the ASE and $\mathrm{EE}$ versus the ratio of $\mathrm{mBS}$ density to $\mathrm{MU}$ density under different ratios of $\mathrm{pBS}$ density to $\mathrm{mBS}$ density, respectively. It can be seen from the figures that (1) when $\lambda_{u}$ is given, for an arbitrary curve, the ASE increases with $\Delta_{1}$ in Figure 2. For a given $\Delta_{1}$, the ASE also increases with $\Delta_{2}$ in Figure 2. This is 
TABLE 1: Parameters used in simulation.

\begin{tabular}{lcc}
\hline Parameters & Notation & Value \\
\hline Area & $S_{\dot{A}}$ & $100 \times 100 \mathrm{~km}^{2}$ \\
Total bandwidth & $B$ & $10 \mathrm{MHz}$ \\
Noise power & $\delta^{2}$ & $-120 \mathrm{dBm}$ \\
Path loss exponent & $\alpha$ & 4 \\
mBS transmit power & $P_{1}$ & $40 \mathrm{~W}$ \\
pBS transmit power & $P_{2}$ & $6 \mathrm{~W}$ \\
mBS static power consumption & $p_{c 1}$ & $400 \mathrm{~W}$ \\
pBS static power consumption & $p_{c 2}$ & $56 \mathrm{~W}$ \\
MU density & $\lambda_{u}$ & $0.01 \mathrm{~m}^{-2}$ \\
Minimum required ASE & $\bar{\eta}_{\text {ASE }}$ & $0.03 \mathrm{bps} / \mathrm{Hz}^{2} / \mathrm{m}^{2}$ \\
SINR threshold & $\theta_{k}$ & $0 \mathrm{~dB}$ \\
\hline
\end{tabular}

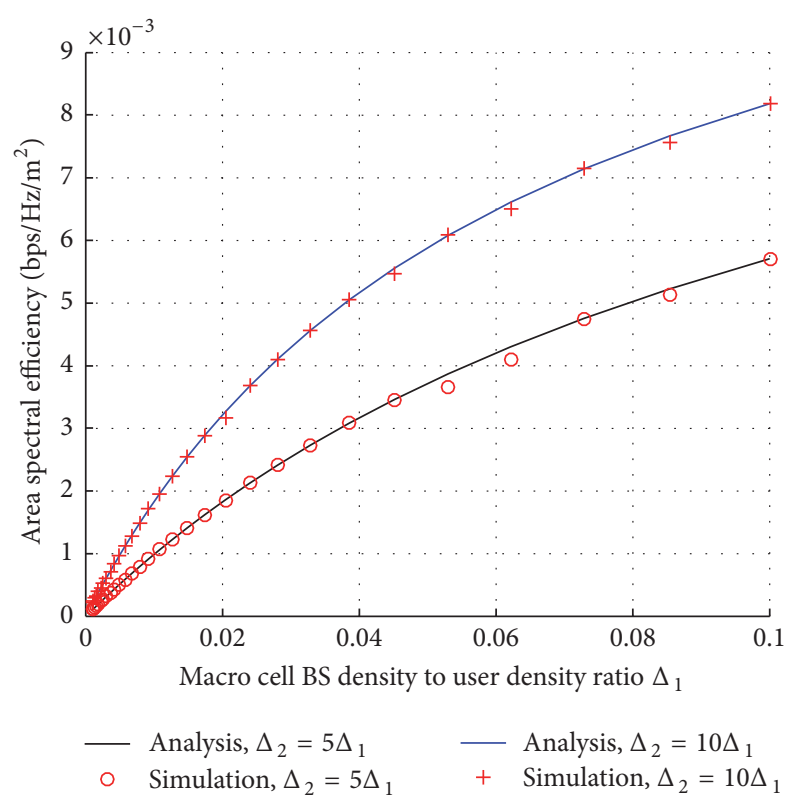

FIGURE 2: ASE versus the ratio of mBS density to MU density.

because when the BS density increases, the network throughput will increase. However, when the BS density surpasses a certain threshold, the ASE will increase slower than before. It is because that increasing BS density will make more interfering BSs close to MU. (2) Figure 3 shows that, for different values of $\Delta_{2}$, the EE increases with $\Delta_{1}$ to a peak and then starts to decrease. As increasing the number of mBSs, the network power consumption will increase. Additionally, the speed of throughput growth is slower than the speed of energy consumption. When $\Delta_{1}$ is given, EE increases with $\Delta_{2}$. The reason is that the total number of MUs served by pBSs will grow with $\Delta_{2}$ under given $\Delta_{1}$, which makes the network gains more throughput. (3) Figures 2 and 3 show that, for different $\Delta_{2} / \Delta_{1}$, different optimal $\Delta_{1}$ and corresponding $\Delta_{2}$ can be found to get maximum EE under the condition of satisfying minimum required ASE.

The impact of pBS density to mBS density under different ratios of mBS density to MU density on the ASE and EE is

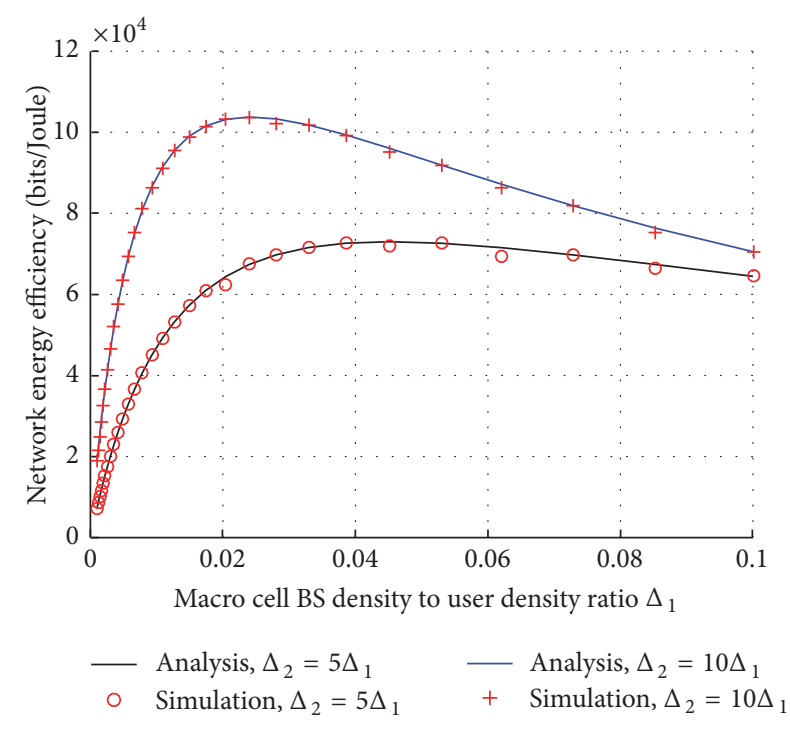

FIGURE 3: EE versus the ratio of mBS density to MU density.

shown in Figures 4 and 5, respectively. The tendency of the ASE shown in Figure 4 and the reason are the same as those in Figure 2. In Figure 5, we can observe that the EE first increases with $\Delta_{2} / \Delta_{1}$ and then decreases under given $\Delta_{1}$. When $\Delta_{2} / \Delta_{1}$ is less than a certain value, for a given $\Delta_{2} / \Delta_{1}$, a larger BS density leads to a greater EE. By contrary, when $\Delta_{2} / \Delta_{1}$ is more than a certain value, for a given $\Delta_{2} / \Delta_{1}$, a larger BS density leads to a smaller EE. The explanation of this result is that when the BS density grows, the total number of BSs is supposed to increase which leads to more power consumption and interfering BSs. Moreover, the energy consumption of a mBS is much more than that of a pBS. In addition, we can conclude that if the MU densities $\lambda_{u}$ and $\Delta_{1}$ are determined, we can find out optimal $\Delta_{2}$ to get maximum EE under the condition of satisfying minimum required ASE.

\section{Conclusions}

In this paper, the effect of BS deployment on the ASE and EE of an ultradense HetNet has been studied. We derived analytical expressions of ASE and EE in terms of the BS density 


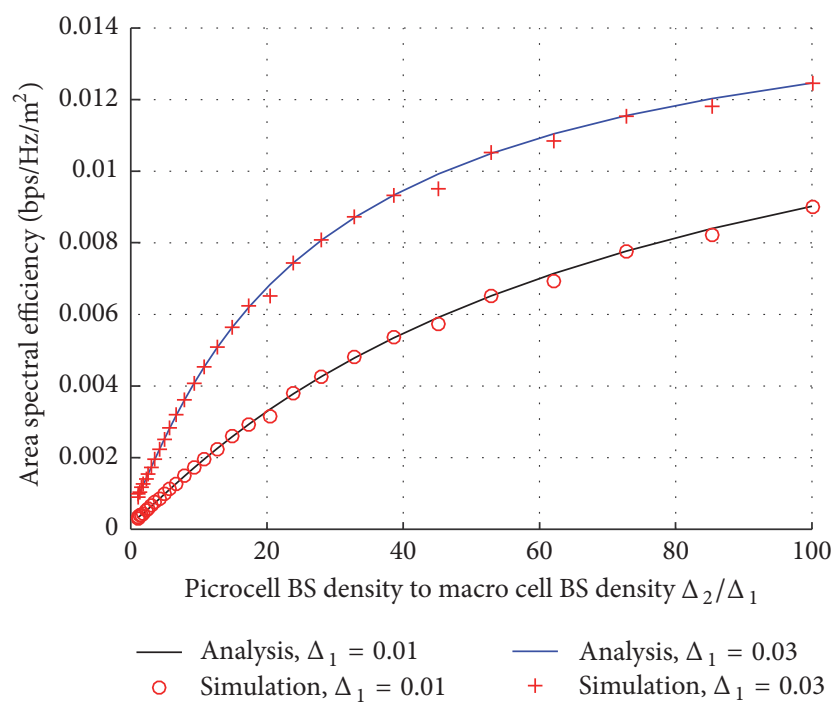

FIGURE 4: ASE versus the ratio of pBS density to mBS.

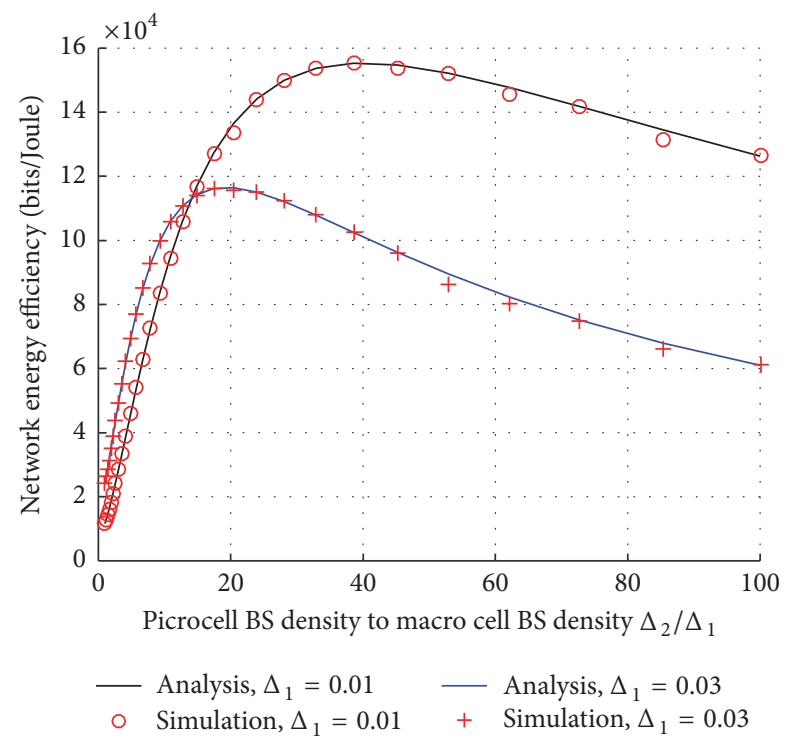

FIGURE 5: EE versus the ratio of pBS density to mBS density.

using the stochastic geometry. Simulation results validated the analysis and showed that the ASE and EE cannot be optimized simultaneously under given system parameters. We need to balance them to achieve a better network performance. Finally, we found out optimal BS densities to maximize the EE under the condition of satisfying the ASE requirement. In addition to ASE and EE, latency and reliability are also important metrics in 5G. Especially in ultradense HetNets, a huge amount of data is transmitted over a large number of access points which will inevitably increase the latency and reduces the reliability to some extent. The tradeoff between them is a very interesting problem that needs to be investigated in the future. Also, the channel state information feedback is very important for managing power resources to balance ASE and EE, which will be investigated later on considering the gain as well as the overhead.

\section{Conflicts of Interest}

The authors declare that they have no conflicts of interest.

\section{Acknowledgments}

This research was supported by the National Natural Science Foundation of China (61671165, 61471135), the Guangxi Natural Science Foundation (2015GXNSFBB139007, 2016GXNSFGA380009), the Fund of Key Laboratory of Cognitive Radio and Information Processing (Guilin University of Electronic Technology), Ministry of Education, China, and the Guangxi Key Laboratory of Wireless Wideband Communication and Signal Processing (CRKL160105), and the Innovation Project of GUET Graduate Education (2016YJCX91, 2017YJCX27).

\section{References}

[1] A. Osseiran, F. Boccardi, and V. Braun, "Scenarios for $5 \mathrm{G}$ mobile and wireless communications: the vision of the METIS project," IEEE Communications Magazine, vol. 52, no. 5, pp. 2635, 2014.

[2] R. Baldemair, E. Dahlman, S. Parkvall, and Y. Selen, "Future Wireless Communications," in Proceedings of the 2013 IEEE 77th Vehicular Technology Conference, pp. 1-5, Spring, Dresden, Germany, June 2013.

[3] G. Auer, V. Giannini, C. Desset et al., "How much energy is needed to run a wireless network?" IEEE Wireless Communications, vol. 18, no. 5, pp. 40-49, 2011.

[4] Y. S. Soh, T. Q. S. Quek, M. Kountouris, and H. Shin, "Energy efficient heterogeneous cellular networks," IEEE Journal on Selected Areas in Communications, vol. 31, no. 5, pp. 840-850, 2013.

[5] Q. Ren, J. Fan, X. Luo, Z. Xu, and Y. Chen, "Analysis of spectral and energy efficiency in ultra-dense network," in Proceedings of the IEEE International Conference on Communication Workshop, ICCW' '15, pp. 2812-2817, June 2015.

[6] Q. Li, G. Wu, and R. Q. Hu, "Analytical study on network spectrum efficiency of ultra dense networks," in Proceedings of the 2013 IEEE 24th Annual International Symposium on Personal, Indoor, and Mobile Radio Communications, PIMRC '13, pp. 2764-2768, September 2013.

[7] Z. Niu, Y. Wu, J. Gong, and Z. Yang, "Cell zooming for costefficient green cellular networks," IEEE Communications Magazine, vol. 48, no. 11, pp. 74-79, 2010.

[8] S. McLaughlin, P. M. Grant, J. S. Thompson et al., "Techniques for improving cellular radio base station energy efficiency," IEEE Wireless Communications, vol. 18, no. 5, pp. 10-17, 2011.

[9] T. Chen, Y. Yang, H. Zhang, H. Kim, and K. Horneman, "Network energy saving technologies for green wireless access networks," IEEE Wireless Communications, vol. 18, no. 5, pp. 3038, 2011.

[10] K. Son, H. Kim, Y. Yi, and B. Krishnamachari, "Base station operation and user association mechanisms for energy-delay tradeoffs in green cellular networks," IEEE Journal on Selected Areas in Communications, vol. 29, no. 8, pp. 1525-1536, 2011. 
[11] T. Zhang, J. Zhao, L. An, and D. Liu, "Energy efficiency of base station deployment in ultra dense HetNets: a stochastic geometry analysis," IEEE Wireless Communications Letters, vol. 5, no. 2, pp. 184-187, 2016.

[12] S. Stefanatos and A. Alexiou, "Access point density and bandwidth partitioning in ultra dense wireless networks," IEEE Transactions on Communications, vol. 62, no. 9, pp. 3376-3384, 2014.

[13] S. Samarakoon, M. Bennis, W. Saad, M. Debbah, and M. LatvaAho, "Ultra dense small cell networks: Turning density into energy efficiency," IEEE Journal on Selected Areas in Communications, vol. 34, no. 5, pp. 1267-1280, 2016.

[14] S. Yunas, M. Valkama, and J. Niemelä, "Spectral and energy efficiency of ultra-dense networks under different deployment strategies," IEEE Communications Magazine, vol. 53, no. 1, pp. 90-100, 2015.

[15] Y. J. Chun, A. Omri, and M. O. Hasna, "Joint optimization of area spectral efficiency and delay over PPP interfered ad-hoc networks," IEEE Communications Letters, vol. 19, no. 10, pp. 1682-1685, 2015.

[16] F. H. Panahi and T. Ohtsuki, "Stochastic geometry modeling and analysis of cognitive heterogeneous cellular networks," EURASIP Journal on Wireless Communications and Networking, vol. 2015, no. 1, 19 pages, 2015.

[17] H. Elsawy, E. Hossain, and M. Haenggi, "Stochastic geometry for modeling, analysis, and design of multi-tier and cognitive cellular wireless networks: a survey," IEEE Communications Surveys \& Tutorials, vol. 15, no. 3, pp. 996-1019, 2013.

[18] Y. J. Chun, M. O. Hasna, and A. Ghrayeb, "Modeling heterogeneous cellular networks interference using poisson cluster processes," IEEE Journal on Selected Areas in Communications, vol. 33, no. 10, pp. 2182-2195, 2015.

[19] Y. Li, F. Baccelli, H. S. Dhillon, and J. G. Andrews, "Statistical modeling and probabilistic analysis of cellular networks with determinantal point processes," IEEE Transactions on Communications, vol. 63, no. 9, pp. 3405-3422, 2015.

[20] N. Deng, W. Zhou, and M. Haenggi, "The ginibre point process as a model for wireless networks with repulsion," IEEE Transactions on Wireless Communications, vol. 14, no. 1, pp. 107-121, 2015.

[21] M. Haenggi, "The meta distribution of the SIR in poisson bipolar and cellular networks," IEEE Transactions on Wireless Communications, vol. 15, no. 4, pp. 2577-2589, 2016.

[22] Y. Zhou, R. Li, Z. Zhao, X. Zhou, and H. Zhang, "On the $\alpha-$ stable distribution of base stations in cellular networks," IEEE Communications Letters, vol. 19, no. 10, pp. 1750-1753, 2015.

[23] F. Baccelli and B. Blaszczyszyn, "Stochastic geometry and wireless networks: volume I theory," Foundations and Trends in Networking, vol. 3, no. 3-4, pp. 249-451, 2009.

[24] D. Stoyan, W. S. Kendall, and J. Mecke, Stochastic Geometry and Its Applications, John Wiley and Sons, New York, NY, USA, 2nd edition, 1996.

[25] S. Singh, H. S. Dhillon, and J. G. Andrews, "Offloading in heterogeneous networks: modeling, analysis, and design insights," IEEE Transactions on Wireless Communications, vol. 12, no. 5, pp. 2484-2497, 2013. 


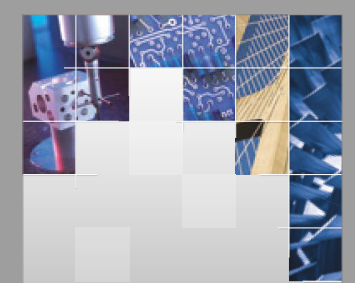

\section{Enfincering}
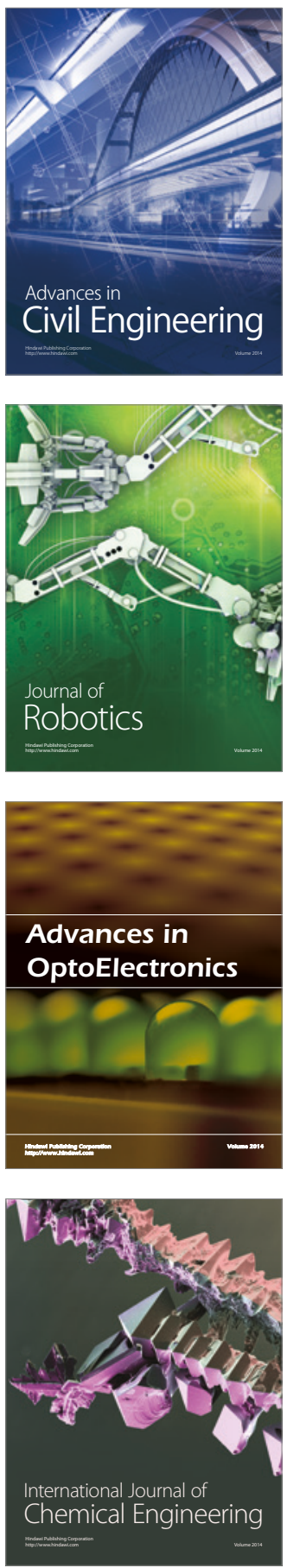

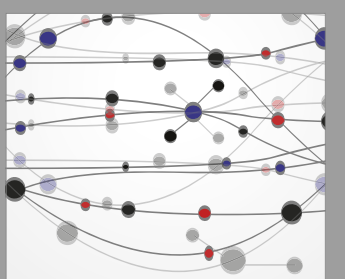

The Scientific World Journal

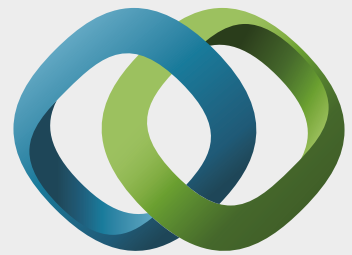

\section{Hindawi}

Submit your manuscripts at

https://www.hindawi.com
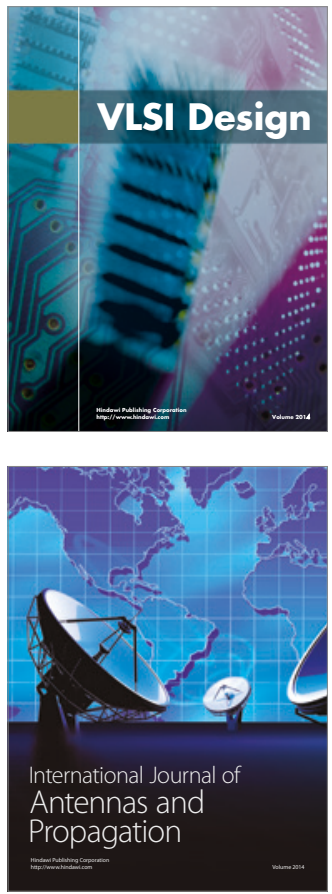

\section{Rotating}

Machinery
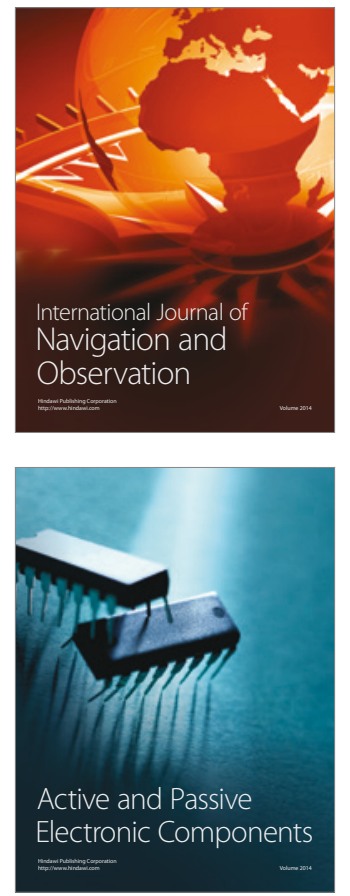
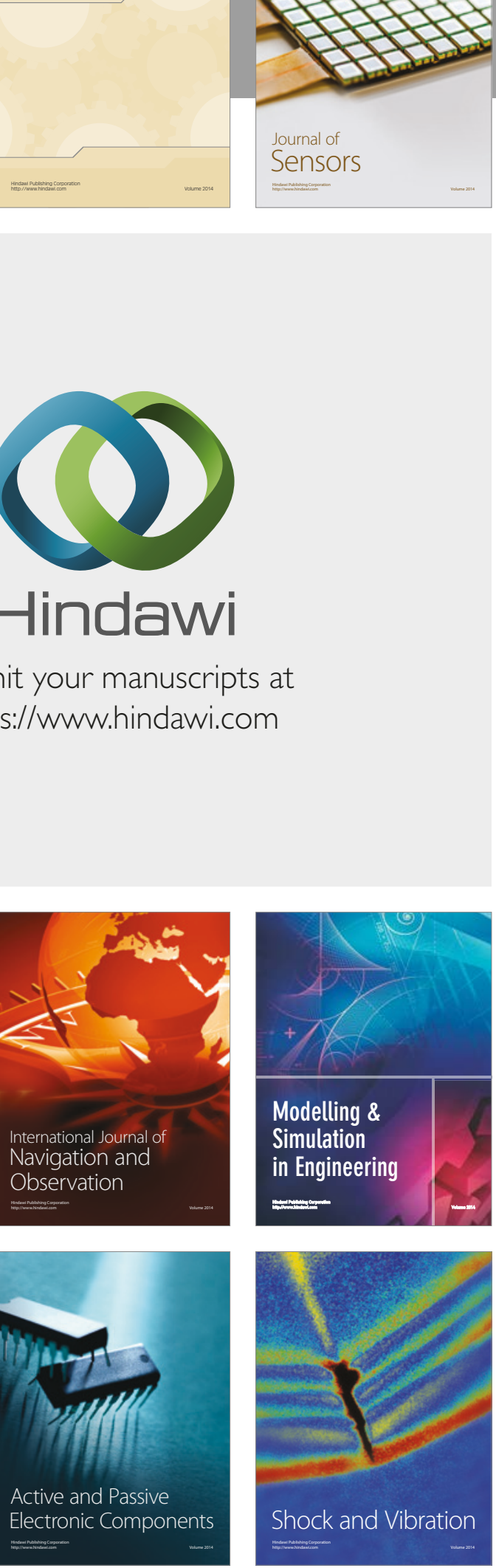
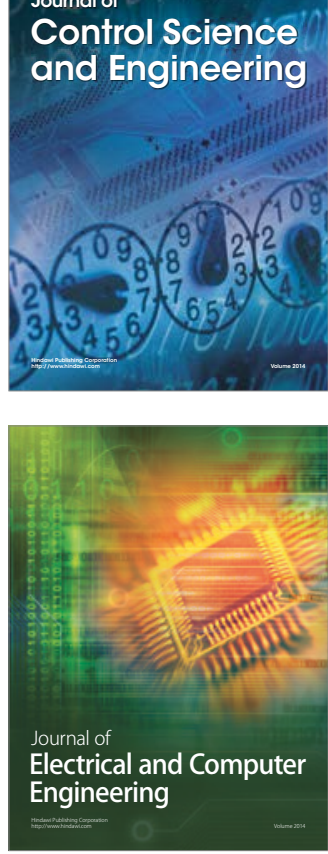

Distributed

Journal of

Control Science

and Engineering
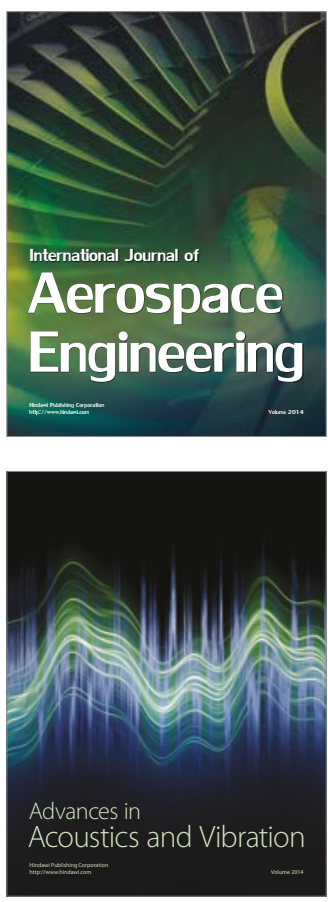

Sensor Networks 